\title{
EMBRYOLOGY OF EXOMPHALOS AND ALLIED MALFORMATIONS*
}

\author{
BY \\ BERNARD DUHAMEL \\ From the Department of Paediatric Surgery, Hôpital de Saint-Denis, Seine, France
}

True congenital malformations, that is to say, those resulting from a defect of the embryonic development and not from a foetal disease, are minor forms of the great monstrosities to which they are related by a continuous series of intermediary forms.

Normal embryology explains monstrosities and thus malformations; anatomic study and the experimental creation of monstrosities allow a better understanding of certain embryogenetic mechanisms.

These notions are admitted for the encephalomyelo-dysplasias (Giroud, Martinet and Solères, 1958), and for malformations of the cephalic extremity, and I have shown that they are also valid for malformations of the caudal extremity (Duhamel, 1961).

Exomphalos belongs to the group of ventral wall malformations that result from a disturbance of the vital mechanism of closing of the body of the embryo.

After the initial segmentation and gastrulation stages, the primitive embryo resembles a flat, oval disk (the germinal disk), the dorsal layer of which, the ectoblast, is continuous with the wall of the amniotic vesicle; the ventral layer, the entoblast, is continuous with the wall of the yolk sac (Figs. $1 \mathrm{~A}$ and $2 \mathrm{~A}$ ).

With the exception of two zones which will remain didermic (the oral membrane and the cloacal membrane), these two cellular layers are separated by a third intermediary layer, the mesoblast, the formation of which occurred during gastrulation, and which constitutes the framework of the future embryo.

This mesoblast will form, in the median plane, the notochord which represents the axial skeleton of the embryo. On each side of the notochord, the para-axial mesoblast will become segmented, forming somites and nephrotomes. In the periphery, the mesoblast stretches without segmentation into the lateral lamina to form the embryonic

* A paper read at a meeting of the British Association of Paediatric Surgeons in London, September 1962. mesenchyme up to the limits of the germinal disk. It then continues as the primary extra-embryonic mesenchyme which separates the two primitive vesicles.

At a very early stage, this primary extra-embryonic mesenchyme condenses at the point of contact with the primitive amnion and yolk sac and divides between them to form a cavity, the extra-embryonic coelom. This condensation and division continues concentrically at the level of the embryonic mesenchyme of the germinal disk, to form the intraembryonic coelom, which is continuous with that of the extra-embryonic coelom. The lateral lamina of the germinal disk are thus divided into a dorsal somatic layer (the somatopleure) which is formed by the condensation of the embryonic mesenchyme at the point of contact with the ectoblast, and into a ventral splanchnic layer (the splanchnopleure) which is formed by condensation of the embryonic mesenchyme at the point of contact with the entoblast (Fig. 1B).

The closing of the body of the embryo, which leads to the formation of the body stalk (Potter, 1952), is due to the considerable growth of the dorsal axis of the embryo (a consequence of the development of the nervous system and of the differentiation of the para-axial mesoblast into somites and nephrotomes).

As it grows, this dorsal axis becomes elevated and as a result the lateral parts of the embryo fold over and form the ventral wall of the embryo (Figs. 1 and 2, C and D). This folding process can be likened to the closing of a purse or, more precisely, to that of two purses, because the lateral laminae are composed of two layers separated by the cavity of the intra-embryonic coelom.

This folding is circumferential (Fig. 3), but four folds may be distinguished as follows (Wolff, 1948).

(1) A cephalic fold whose splanchnic layer containing the outline of the heart and the large blood vessels will close the foregut in front. Its somatic layer will form the thoracic and epigastric wall as well as the septum transversum (Fig. $2 \mathrm{C}$ and $\mathrm{D}$ ).

(2) A caudal fold, whose splanchnic layer will 


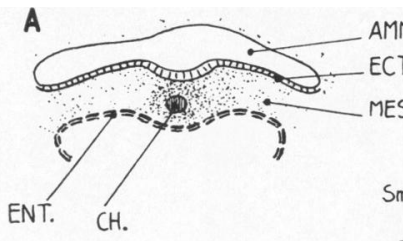

FiG. 1.-Transversal schematic sections of human embryos of $1 \cdot 5,2 \cdot 0,2 \cdot 5$ and $3 \mathrm{~mm}$., approximately.

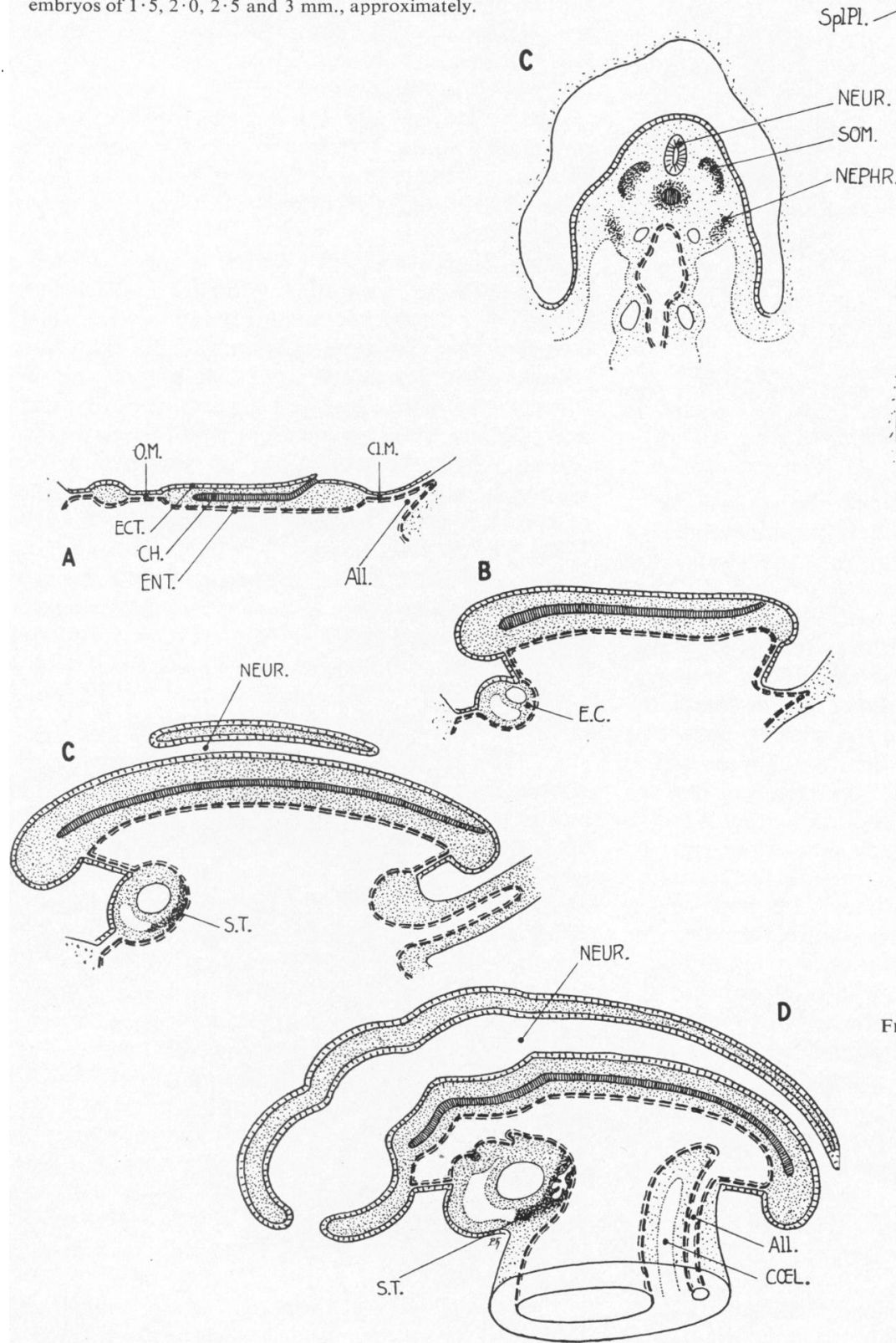

CT.

B
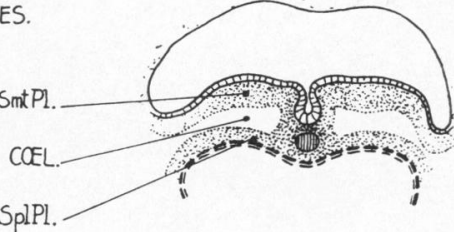

FIG. 2.-The same embryos in sagittal schematic sections. 


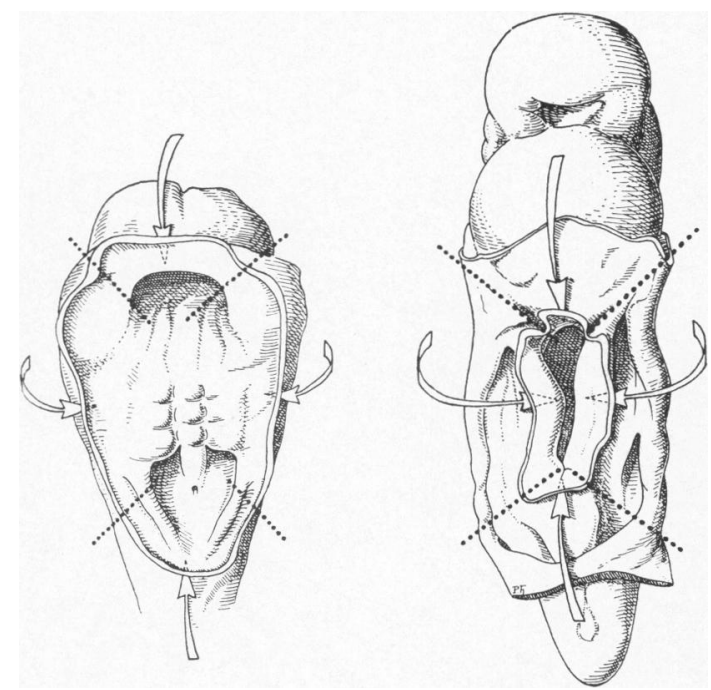

FIG. 3.-Ventral views of human embryos of 2 and $3 \mathrm{~mm}$. The body stalk is sectioned near the future umbilical ring. The arrows indicate the direction of folding. The dotted lines indicate the arbitrary limit of the embryonic folds.

close the hindgut, in front, and the somatic layer including the allantois, which is the forerunner of the urinary bladder which will form the hypogastric wall (Fig. 2D).

( 3 and 4) Lateral folds which close the midgut and form the lateral walls of the abdomen (Fig. 1D).

The apex of these folds is the future umbilical ring. Here the splanchnic layer which limits the cavity of the primitive gut in the embryo protrudes with the wall of the yolk sac through an orifice which later will elongate to become the vitelline duct. The somatic layer of the folds extends to the wall of the amniotic sac which now surrounds the embryo. The cavity of the intra-embryonic coelom communicates for some time with the extraembryonic coelom. In later embryonic life the extra-embryonic coelom will gradually disappear.

Once the body of the embryo is closed and the simultaneous development of the nervous system and of the extremities has been completed the embryo has assumed its final form; morphogenesis is completed.

Teratogenic actions that occur during this period may inhibit one or several of these morphogenetic processes, but will not prevent secondary tissue differentiation (Wolff, 1948). They will produce an abnormal morphology or, in other words, a monstrosity.

Subsequently, the mesoblastic outlines will continue their evolution to form the various organs.
This is the organogenetic stage. The teratogenic actions which now occur will inhibit the differentiation of the mesoblastic outlines and will thus produce malformations.

Differentiation of the mesoblast varies in the different regions of the embryo. The axial mesoblast forms the notochord which in the human is a transitory organ. It determines the differentiation of the dorsal ectoblast into nervous tissue, the differentiation of mesoblast into somites, nephrotomes and lateral lamina, and of the ventral entoblast into the intestinal tube.

The para-axial mesoblast is segmented into somites and nephrotomes. These mesoblastic masses differentiate to form the axial skeleton, the dorsal musculature and the internal urinary and genital systems.

The mesoblast of the lateral lamina does not segment. It is not solid buit forms a soft cellular tissue, the embryonic mesenchyme, which is the framework of the somatopleure and the splanchnopleure. It differentiates in some places and will form (1) the ventral wall and the outline of the limbs, and (2) the visceral muscles and the circulatory system. This differentiation is induced by the para-axial mesoblast and not by ventral extension of the somites, as is the common belief (Wyburn, 1937).

The inhibition of the morphogenetic process of the closing of the body of the embryo causes a series of malformations called celosomias (Geoffroy SaintHilaire, 1836), i.e. herniae of the abdominal wall or ventral herniae. They are due to a failure of formation of all or part of the embryonic folds.

1. The failure of formation of the cephalic fold seldom influences the splanchnic layer of the fold, as the latter contains the heart and the large blood vessels without which the embryo cannot develop (the frequent occurrence of major cardiovascular malformations in the celosomias, especially in cases with cephalic fold involvement, should be noted). Early failure of formation of the somatic layer of the cephalic fold causes an upper celosomia (Fig. 4), in which the thoracic and epigastric wall is missing resulting in an ectopia cordis, with anterior sternal and diaphragmatic defect and an exomphalos (Cantrell, Haller and Ravitch, 1958). Late inhibition of this process will prevent the septum transversum from reaching the posterior vertebral plane and persistent pleuro-peritoneal canals with diaphragmatic herniae will result. Abdominal ectopia of the heart is often associated with exomphalos (Duhamel, 1953).

2. Failure of the formation of the caudal fold can affect the somatic layer as well as the splanchnic 
layer. Failure of formation of the splanchnic layer causes a partial agenesis of the hindgut which opens into the bladder (Trusler, Mestel and Stephens, 1959). Failure of formation of the two layers leads to a lower celosomia (Duhamel, quoted by Quetard, 1961) (Fig. 5), comprising an exomphalos, agenesis of the hindgut and a fistula between the intestine and an ectopia vesicae (Singer, 1959; Uson, Lattimer and Melicow, 1959; Rickham, 1960; Soper and Green, 1961). This monstrosity should not be called a 'cloacal exstrophy' as it does not implicate the cloaca, but rather the caudal fold of the embryo; its embryology has been described by Johnston (1913). It is probable that partial failure of formation of the caudal fold is connected with the agenesis of one of the umbilical arteries, frequently associated with malformations of the ventral wall. Bourne and Benirschke (1960) thought that agenesis of an umbilical artery might, in fact, cause a malformation of the caudal fold; it seems more likely that the agenesis is the result of the caudal fold malformation.

Local failure of formation of the somatic layer results in absence of the hypogastric wall in front of the allantois. This explains the common association of exstrophy of the urinary bladder with exomphalos. Only in exceptional cases is it associated with malformations of the uro-genital sinus.

3. Failure of formation of the lateral folds usually affects only the somatic layer of the folds. It seems that the entoblast of the splanchnic layer forming the midgut is not very sensitive to teratogenic actions (Ancel, 1950; Buck, Clavert and Rumpler, 1962). Failure of embryonic folding at the level of the lateral folds prevents the body from closing completely, and the umbilical orifice remains widely open thus causing a middle celosomia, in other words, an exomphalos (see Table).

The somatopleure continues with the amniotic wall and the intra-embryonic coelom communicates widely with the remainder of the extra-embryonic coelom. This large cavity is occupied by the midgut which is frequently incompletely rotated.

TABLE

30 PERSONAL CASES OF EXOMPHALOS

\begin{tabular}{|c|c|c|}
\hline \multicolumn{2}{|r|}{ Exomphalos } & No. \\
\hline Upper celosomia & $\left\{\begin{array}{l}\text { Exomphalos, defect of sternum, dia- } \\
\text { phragm, pericardium and heart } \\
\text { Exomphalos, diaphragmatic hernia and } \\
\text { ectopia cordis abdominalis } \\
\text { Exomphalos and diaphragmatic hernia } \ldots\end{array}\right.$ & $\begin{array}{l}0 \\
2 \\
2\end{array}$ \\
\hline Middle celosomia & Exomphalos alone $\quad \%$ & 24 \\
\hline Lower celosomia & $\begin{array}{l}\text { Exomphalos and bladder exstrophy } \\
\text { Exomphalos, hindgut agenesis, vesico- } \\
\text { intestinal fissure and bladder exstrophy }\end{array}$ & $\begin{array}{l}1 \\
1\end{array}$ \\
\hline
\end{tabular}

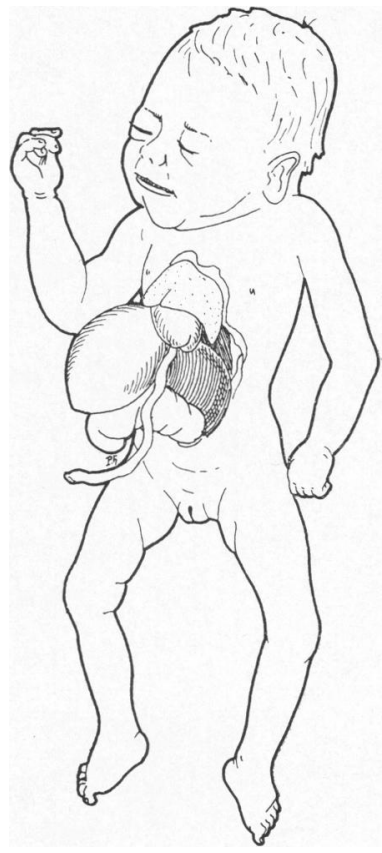

FIG. 4.-Upper celosomia.

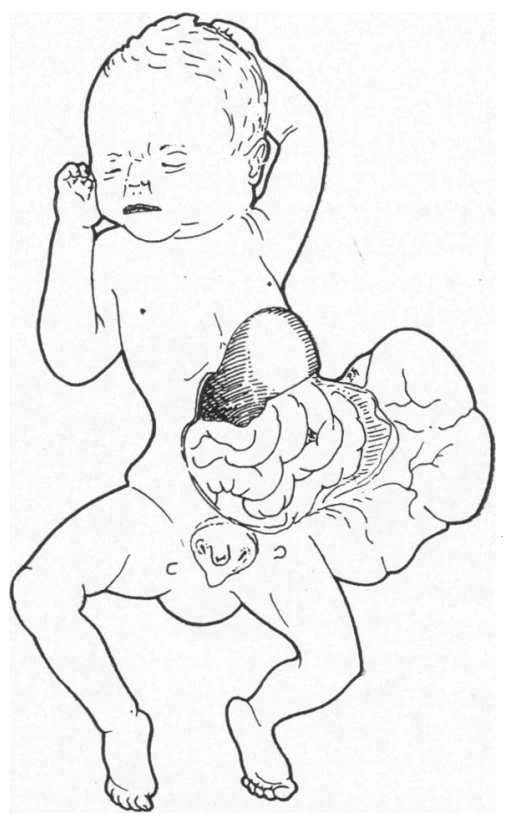

FIG. 5.-Lower celosomia. 


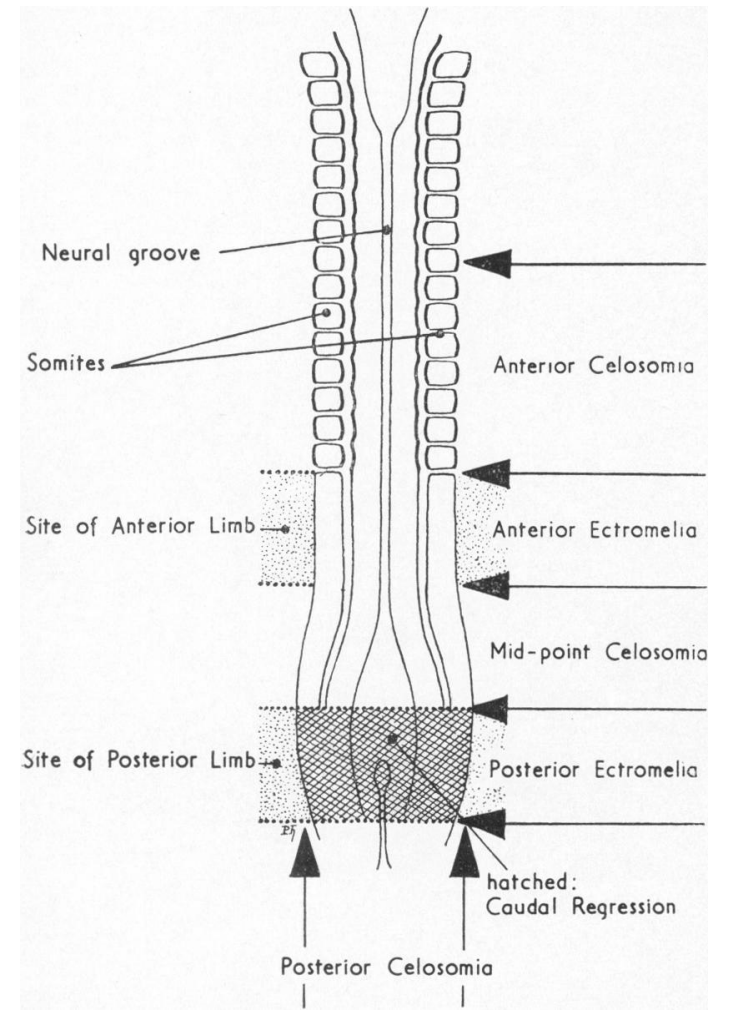

FIG. 6.-Schematic representation of the zones where it is possible to obtain specific malformations by direct teratogenic action on the chick embryo at the 15-somites stage (according to Wolff).

The embryonic part of this hernia is developed from the somatopleure and will form the abdominal wall which has a normal anatomic structure. The primary extra-embryonic mesenchyme remains undifferentiated, mucoid and avascular.

There is no defect of mesodermization of an amniotic wall as this never differentiates; neither is there persistence of a physiological hernia because the latter does not result in defective closing of the umbilical ring but rather in a delayed obliteration of the extra-embryonic coelom. It can, therefore, only result in a hernia of the cord. Moreover, as Bremer (1957) has indicated, the physiological hernia contains only midgut and never the liver or the other viscera which are so often present in an exomphalos. The prolapse of the intestinal loops into the exomphalos is the consequence rather than the cause of the malformation (Ancel, 1950). In the hernia of the cord, as in an ordinary infantile inguinal hernia, the partial defect is of no importance; the only important factor is the persistence of a peritoneal diverticulum.
It should also be pointed out that the formation of the intra-embryonic coelom by division of the splanchnopleure and the somatopleure is a very early event which precedes the closing of the embryo. Thus, it is not possible that embryonic adhesions between the midgut and the pouch of an exomphalos will occur; adhesions can only occur as a result of secondary foetal accidents (A. Giroud, personal communication).

All these embryonic observations, as well as those which can be made during operations (Duhamel, 1953), are a valid argument against the old classification of exomphalos into embryonic forms and foetal forms.

Exomphalos is thus the result of a failure of morphogenesis; it is, therefore, a monstrosity.

Many monstrosities resulting from inhibition of embryonic folding can be produced experimentally. Bremer (1928) and Wolff (1936) were successful in reproducing the different varieties of celosomia in chick embryos. Wolff has mapped out the effect of direct teratogenic actions (Fig. 6); he also explains how diffusion of the teratogenic action can produce teratological associations in adjoining parts. Diffusion can be directed laterally and then affect the limbs; it can be directed medially and affect the nephrotomes, causing agenesis or malformation of the kidneys, or the somites themselves, causing malformation of the spinal skeleton. The neural tube may be affected, especially in the lower celosomia where, as I have stated, even an extensive malformation may not necessarily be fatal. In these cases a sacral meningocele is frequently observed. My assistant, Soymie (1960), has described many examples of these teratological associations in adjoining parts, in his thesis.

As we have already seen, later teratogenic actions during the organogenetic stage cause malformations. Here, the overall appearance of the embryo is preserved; there is no exomphalos. The essential phenomenon lies in failure of differentiation of the mesoblastic outlines of the different organs.

A relatively early teratogenic action may prevent the differentiation of the embryonic mesenchyme which forms the framework of the somatopleure. The ectoblastic layer of the somatopleure, deprived of its mesenchymal support, will be resorbed in the course of intrauterine life, as are the oral and cloacal membranes, while the amniotic wall of the exomphalos which contains a specific extra-embryonic mesenchyme, Wharton's jelly, will not resorb during intrauterine life.

This type of malformation when occurring in the region of the lateral fold is called gastroschisis 
(Moore and Stokes, 1953; Kiesewetter, 1957; Berman, 1957; Cook, 1959); it seems preferable to call it para-omphalocele (Lotte, 1959). This malformation can be distinguished from exomphalos by its position, which is always lateral to the umbilicus, and by the absence of amniotic coverings.

In the region of the cephalic fold, the most frequent malformation is ectopia cordis, in which the heart is exposed, lying in a defect of the anterior thoracic wall (Friedlieb and McDonald, 1950; Hurwitt and Lebendiger, 1959).

In the region of the caudal fold, the resorption of the somatic layer produces an exstrophy of the urinary bladder which, as we now know, is caused by malformation of the caudal fold and not by a defect of the cloacal membrane.

The most delayed teratogenic actions will only prevent differentiation of the somatic layers of the mesenchyme. They can cause an agenesis of the skeleton, in particular of the sternum (Rehbein and Hofmann, 1961), or of the ribs (Rickham, 1959), or a muscle agenesis, especially of the muscles of the abdominal wall (Boissonnat and Duhamel, 1962). Finally, sometimes the differentiation of the skeleton and muscles is normal, but the skin may be incompletely formed causing limited skin aplasias (Boureau, 1961), similar to those that can be found in the region of the posterior nervous fusion (Giroud and Roux, 1961).

\section{REFERENCES}

Ancel, A. (1950). La Chimiotératogenèse. Doin, Paris.

Berman, E. J. (1957). Arch. Surg., 75, 788.

Boissonnat, P. and Duhamel, B. (1962). Brit. J. Urol., 34, 59.

Boureau, M. (1961). Presse méd., 69, 2135 and 2198.

Bourne, G. L. and Benirschke, K. (1960). Arch. Dis. Childh., $35,534$.

Bremer, J. L. (1928). Anat. Rec., 37, 225.

(1957). Congenital Anomalies of the Viscera. Harvard University Press, Cambridge.

Buck, P., Clavert, J. and Rumpler, Y. (1962). Ann. Chir. infant., 3, 73 .

Cantrell, J. R., Haller, J. A. and Ravitch, M. M. (1958). Surg. Gynec. Obstet., 107, 602.

Cook, T. D. (1959). Surgery, 46, 618.

Duhamel, B. (1953). Chirurgie du nouveau-né et du nourrisson. Masson, Paris. (1961). Arch. Dis. Childh., 36, 152.

Friedlieb, O. and McDonald, J. J. (1950). Surgery, 28, 864.

Geoffroy Saint-Hilaire, I. (1836). Histoire générale et particulière des anomaties. Baillière, Paris.

Giroud, A., Martinet, M. and Solères, M. (1958). Rev. neurol., 98 181.

- and Roux, C. (1961). Bull. Soc. franc. Derm. Syph., 68, 197.

Hurwitt, E. S. and Lebendiger, A. (1959). Arch. Surg., 78, 197.

Johnston, T. B. (1913). J. Anat. Entwickl., 55, 201.

Kiesewetter, W. B. (1957). Arch. Surg., 75, 28.

Lotte, J. (1959). Ann. Chir. plast., 4, 156.

Moore, T. C. and Stokes, G E. (1953). Surgery, 33, 112

Potter, E. L. (1952). Pathology of the Fetus and the Newborn.

Year Book Publishers, Chicago.
Quetard, R. H. (1961). Thèse de la Faculté de Médecine de Paris.

Rehbein, F. and Hofmann, G. (1961). Chirurg, 32, 106.

Rickham, P. P. (1959). Arch. Dis. Childh., 34, 14.

(1960) ibid., 35, 97.

Singer, H. (1959).' Zbl. Chir., 84, 1752.

Soper, R. T. and Green, E. W. (1961). Surg. Gynec. Obstet., 113, 501. Soymie, J. C. (1960). Thèse de la Faculté de Médecine de Paris.

Trusler, G. A., Mestel, A. L. and Stephens, C. A. (1959). Surgery, 45, 328.

Uson, A. C., Lattimer, J. K. and Melicow, M. M. (1959). Pediatrics, $23,927$.

Wolff, E. (1936). Arch. Anat. (Strasbourg), $22,1$.

- (1948). La Science des monstres. Gallimard, Paris.

Wyburn, G. M. (1937). J. Anat. (Lond.), 71, 201. 